\title{
Screening of Melon Populations for Resistance to Didymella bryoniae in Greenhouse and Plastic Tunnel Conditions
}

\author{
Cláudio Yuji Tsutsumi ${ }^{1 *}$ and Norberto da Silva ${ }^{2}$ \\ ${ }^{1}$ Centro de Ciências Agrárias; UNIOESTE; Rua Pernambuco, 1777; 85960-000; Marechal Cândido Rondon - \\ PR - Brazil. ${ }^{2}$ Departamento de Produção Vegetal; Setor de Agricultura e Melhoramento Vegetal; FCA; UNESP; \\ C. P. 237; 18603-970, Botucatu - SP - Brazil
}

\begin{abstract}
Studies were carried out to evaluate melon populations for resistance to Gummy Stem Blight, caused by Didymella bryoniae for use in breeding programs under greenhouse and plastic tunnel conditions. Were evaluated reactions of half-sib populations of PI 140471 and Anô $n^{\circ} 2$ had as with susceptible check Eldorado 300. Plants were inoculated 45 days for needle prickling followed by application of $0.05 \mathrm{ml}$ of $5.0 \times 10^{5}$ spores $/ \mathrm{ml}$ suspension in axis of third and fourth true leaves.Resistance evaluation was made after 90 days of age using a scale varying one to five. PI 140471 and Anô $n^{o} 2$ progenies were found superior and differed significantly with the checker Eldorado 300 for the $F$ Test. Anô $n^{o} 2$ possessing multiple resistance to diseases and desirable commercial characteristics, constituted the best resistance source to be used in breeding programs.
\end{abstract}

Key words: Cucumis melo, gummy stem blight, plant breeding, disease resistance

\section{INTRODUCTION}

Gummy Stem Blight, caused by Didymella bryoniae (Auersw.) Rehm., is considered a disease of increase importance in melon culture, due to intense commercial exploration of these cucurbit. The fungus can infect any tissue and in any stadium of development of plant, showing a great variety of symptoms as leaves stains, stems canker, decay of plant and black rottenness of fruits (Chiu and Walker, 1949). The control of $D$. bryoniae has been made through use of fungicides. However, this method has not been presenting satisfactory results due to resistance developed by fungus after short period of use of these fungicides (Malathrakis and Vakalounakis, 1983). Consequently, the ideal method for the pathogen control is development of resistant varieties. None of the commercially sowed varieties in Brazil presents resistance to gummy stem blight.

Sowell et al. (1966) reported a high resistance level in PI 140471, a native wild melon of Texas, compatible with commercial types of melon. Norton and Prasad (1967) found that the resistance of PI 140471 was controlled by a pair of genes, $M c$ $m c$. Other investigations demonstrated a moderate resistance in the improved lines $\mathrm{C \# 1}$ and $\mathrm{C \# 8}$ of melon Auburn Cantaloup, controlled by a pair of independent genes denominated $M c^{l} m c^{l}$.

Sowell (1981) observed that the resistance of PI 140471 was not shown appropriate in places where disease was severe, and introduced PI 266935 and PI 436533, whose resistance degrees didn't differ of PI 140471. These introductions were shown highly resistant in field tests and greenhouse. Zhang et al. (1997) in greenhouse and field

* Author for correspondence 
evaluations of melon for resistance to gummy stem blight, observed consistent results between the optimized greenhouse screening procedure and inoculated replicated field tests. Takada et al. (1980) identified sources considered resistant: Maekawanakate-shirokawa, Numame, Kankoku n ${ }^{\circ}$ 1, Shirouri-nigô and Mi-tang-ting. Takada (1983) developed resistant varieties from melon to $D$. bryoniae starting from the incorporation of the original resistance of Mi-tang-ting, obtaining Anô $n^{\circ} 1$, Anô $n^{\circ} 2$ and Anô $n^{\circ} 3$. Zanuga et al. (1999) reported two melon accessions, PI 157082 and PI 511890 to be resistant to gummy stem blight. Resistance in both accessions was due to a single dominant gene, PI 140471 possessed different resistance genes.

The present work had the objective of evaluating populations for resistance to $D$. bryoniae for use in breeding programs of melon in greenhouse and plastic tunnel conditions.

\section{MATERIAL AND METHODS}

The work was carried out in two experimental areas of Faculdade de Ciências Agronômicas UNESP, Campus of Botucatu - SP: in the greenhouse at Lageado Experimental Station and in the plastic tunnel at São Manuel Experimental Station were tested.

Half-sib progenies of PI 140471 and Anô $n^{\circ}$ 2, these were obtained from the Departamento de Produção Vegetal - Setor de Agricultura e Melhoramento Vegetal, FCA/UNESP. Commercial variety Eldorado 300 (Asgrow Sementes do Brasil Ltda.) was used as susceptible check. The sowing was done in trays of PVA of 128 alveoli, containing sterilized substrate, constituted of similar parts of soil, sands and bovine manure, suplemented with $5 \mathrm{~g}$ of fertilizer (4-14-8 N-P $\mathrm{O}_{2}-\mathrm{O}_{2} \mathrm{O}$ ) for each liter of substrate. Two seeds were placed per alveolus and the trays were maintained in greenhouse.

In the greenhouse, the obtained seedlings were transplanted (25 days of age) in plastic pots with capacity for one liter of soil. Statistical design used was the entirely randomized design, with 10 to 12 replications. In the plastic tunnel the same were transplanted for the definitive hotbeds, using the experimental design of blocks with two replications. Each experimental parcel consisted of one double row of plants spaced $0.50 \mathrm{~m}$ and spaced $1.00 \mathrm{~m}$ to the adjacent rows. The spacing among plants inside the row was $0.40 \mathrm{~m}$, totaling a maximum of 10 plants and a minimum of six plants per experimental parcel.

For inoculation isolate I-47 was used, originating from Presidente Alves District, in São Paulo State. $D$. bryoniae was grown in autoclaved Azuki (Phaseolus angularis Kaw.) medium maintained at $\pm 24{ }^{\circ} \mathrm{C}$ under constant near ultraviolet light (black light). Suspensions of spores (seven days) were obtained with sterile water and gauged for $5.0 \mathrm{x}$ $10^{5}$ spores $/ \mathrm{ml}$.

The inoculation was accomplished to the 45 days of age of the plants using needle prickling with application of $0.05 \mathrm{ml}$ of suspension of spores in the insertion of third and fourth true leaves. In the greenhouse after inoculation these were maintained in humid enviromment, with aid of transparent plastic film, for a period of 48 hours. After this period, the covering was removed and the plants maintained in humid enviroment. In plastic tunnel the plants were maintained humid with the irrigation aid for furrow.

During the experimental period, plants were evaluated every five days until that checker reached same or superior to mean value to 4.5 , using the disease index: 0 - without symptoms; 1 small lesions growing in the stem or in the leaves; 2 - moderate lesions growing in the stem or in the leaves; 3 - severe lesions growing in the stem or in the leaves; 4 - plant decay, with lesions taking to the subsequent death; 5 - death of the plant.

After the transformation of values in $\sqrt{X+0.5}$, the variance analysis for resistance in function of the time was made, in accordance with Pimentel Gomes (1987). The experimental design was parcels subdivided in the time, with number different from plants inside of each experimental parcel. To detect differences among means the Tukey test was used.

\section{RESULTS AND DISCUSSION}

\section{Evaluation in greenhouse conditions}

The variance analysis of half-sib melon progenies function of the time in greenhouse tested to as level of mean squares and respective significances for test $\mathrm{F}$ are shown in the Table 1. For the test $\mathrm{F}$ to $5 \%$ of probability, the mean squares of progenies, time and the interaction time $x$ progenies were significant. The coefficients of variation was of medium precision. The reactions 
of 12 half-sib progenies of PI 140471 and 15 halfsib progenies of Anô $n^{\circ} 2$ and the susceptible check, Eldorado 300, tested in different times are shown in the Table 2.

The reaction of inoculated plants differed significantly in function of evaluation times. As evaluations moved forward, the severity of symptoms increase progressively, and stabilized in the last evaluation. Significant differences were not observed among populations in evaluations accomplished 30 days after inoculation, just a quick increase in mean severity of the disease in relationship the previous evaluations. The differences between the PI 140471 and Anô $n^{\circ} 2$ progenies and the susceptible checker, Eldorado 300 , showed larger intensity after 35 days of inoculation. Among tested progenies, progeny PI 140471-05 was most resistant, differing significantly with others 30 days after the inoculation. This progeny maintained that difference until the end of evaluations when Eldorado 300 presented $100 \%$ of died plants.

\section{Evaluation in plastic tunnel conditions}

The variance analysis of half-sib progenies in function of time in plastic tunnel, appraised to level of mean squares and respective significances for test $\mathrm{F}$ are shown in the Table 3. For test $\mathrm{F}$ to $5 \%$ of probability mean squares of progenies, time and interaction treatment $\mathrm{x}$ time were significant. The coefficient of variation was considered of high precision.

The reactions of 12 half-sib progenies of PI 140471 and 15 half-sib progenies of Anô No. 2 and the susceptible check, Eldorado 300, appraised in different times are shown in the Table 4. The differences among the half-sib progenies of PI 140471 and Anô $\mathrm{n}^{\circ} 2$ and the susceptible check,
Eldorado 300, became significant 35 days after the inoculation. Before this period the differences were not clear.

The variance analysis, excluded the checker done in the last evaluation (45 days after inoculation), mean squares and respective significances to $5 \%$ of probability are shown in the Table 5. The significance of interaction among means of progenies of two tested populations demonstrated that original progenies of Anô $\mathrm{n}^{\circ} 2$ were superior to the original progenies of PI 140471, with mean values of 2.16 and 2.32 , respectively.

The non-significance of mean square among progenies of Anô $n^{\circ} 2$, indicated uniformity of this variety for reaction to $D$. bryoniae, unlike the PI 140471, where no uniform was shown. The population of melon PI 140471 was described originally by Sowell et al. (1966) as almost immune to $D$. bryoniae when cultivated in field conditions. This population, in the conditions of present experiment, presented a high resistance degree in plastic tunnel. However, resistance was shown unsatisfactory in the greenhouse, which agreed with observations of Sowell (1981) on the increase of the amount of disease under extreme conditions. Anô $\mathrm{n}^{\circ}$ 2, developed in Japan by Takada (1983), behaved in way similar to the PI 140471. The resistance source used in development of Anô $\mathrm{n}^{\mathrm{o}} 2$ was originated of Mitang-ting variety, native of China. That totally differed with the PI 140471, not only with relationship to the origin but also with relationship to group one that belonged. While the PI 140471 is a wild population found in Texas, Mi-tang-ting belongs to group of oriental melons, classified as C. melon var. conomom.

Table 1 - Variance analysis of the resistance of melon progenies inoculated with D. bryoniae in function of the evaluation times in greenhouse conditions. Botucatu, 1992.

\begin{tabular}{lrr}
\hline \multicolumn{1}{c}{ SV } & DF & MS \\
\hline Progenies & 27 & $0.514^{*}$ \\
Error (a) & 301 & 0.109 \\
\hline Parcel & 328 & $18.197^{*}$ \\
Time & 8 & $0.061^{*}$ \\
Time x Progenies & 216 & 0.021 \\
Error (b) & 2408 & 17.28 \\
\hline CV $(\%)$ & & \\
\hline
\end{tabular}

* Significant for the test $\mathrm{F}$ at the level of $5 \%$ of probability. 
Table 2 - Reaction of melon progenies inoculated with D. bryoniae, in function of the evaluation times in greenhouse conditions. Botucatu, 1992.

\begin{tabular}{|c|c|c|c|c|c|c|c|c|c|c|c|c|c|c|c|}
\hline \multirow{2}{*}{ Progenies } & \multicolumn{15}{|c|}{ Evaluation Time $(\text { days })^{1,2}$} \\
\hline & & 5 & & 10 & & 15 & 20 & & 25 & 30 & & 35 & & 40 & 45 \\
\hline Eldorado 300 & 0.35 & $\mathrm{Ea}$ & 2.26 & $\overline{\mathrm{Da}}$ & 2.66 & $\mathrm{CDa}$ & $3.00 \mathrm{BCa}$ & 3.53 & $\mathrm{Ba}$ & $4.28 \mathrm{ABa}$ & 4.79 & $\mathrm{Aa}$ & 4.83 & $\mathrm{Aa}$ & $5.00 \mathrm{Aa}$ \\
\hline PI 140471 - 01 & 0.75 & $\mathrm{Ca}$ & 1.88 & $\mathrm{Ba}$ & 2.32 & $\mathrm{ABa}$ & $2.57 \mathrm{Abc}$ & 2.69 & Aab & $2.77 \mathrm{Ab}$ & 2.92 & $\mathrm{Ab}$ & 2.96 & $\mathrm{Ab}$ & $2.95 \mathrm{Ab}$ \\
\hline PI 140471 - 02 & 0.33 & $\mathrm{Ca}$ & 2.00 & $\mathrm{Ba}$ & 2.20 & $\mathrm{Ba}$ & $2.44 \mathrm{Bbc}$ & 2.53 & $\mathrm{ABbc}$ & $2.61 \mathrm{ABb}$ & 2.74 & $\mathrm{Ab}$ & 2.65 & $\mathrm{Ab}$ & $2.73 \mathrm{Ab}$ \\
\hline PI 140471 -04 & 0.76 & $\mathrm{Ca}$ & 1.96 & $\mathrm{Ba}$ & 2.33 & $\mathrm{ABa}$ & $2.38 \mathrm{ABbc}$ & 2.53 & $\mathrm{Abc}$ & $2.54 \mathrm{Ab}$ & 2.63 & $\mathrm{Ab}$ & 2.69 & $\mathrm{Ab}$ & $2.88 \mathrm{Ab}$ \\
\hline PI 140471 - 05 & 0.51 & $\mathrm{Ca}$ & 0.97 & $\mathrm{Ba}$ & 1.94 & $\mathrm{Ab}$ & $2.10 \mathrm{Ab}$ & 2.10 & Ac & $2.10 \mathrm{Ac}$ & 2.29 & $\mathrm{Ac}$ & 2.38 & $\mathrm{Ac}$ & $2.49 \mathrm{Ac}$ \\
\hline PI 140471-07 & 1.44 & $\mathrm{Ca}$ & 2.11 & $\mathrm{Ba}$ & 2.24 & $\mathrm{Ba}$ & $2.32 \mathrm{ABbc}$ & 2.49 & $\mathrm{ABbc}$ & $2.61 \mathrm{ABb}$ & 2.86 & $\mathrm{Ab}$ & 3.02 & $\mathrm{Ab}$ & $2.98 \mathrm{Ab}$ \\
\hline PI 140471 - 10 & 1.19 & $\mathrm{Ca}$ & 2.14 & $\mathrm{Ba}$ & 2.69 & $\mathrm{ABa}$ & $2.77 \mathrm{ABbc}$ & 2.85 & Aabc & $3.02 \mathrm{Ab}$ & 3.02 & $\mathrm{ABb}$ & 3.06 & $\mathrm{Ab}$ & $3.11 \mathrm{Ab}$ \\
\hline PI 140471 - 11 & 0.64 & $\mathrm{Ca}$ & 2.04 & $\mathrm{Ba}$ & 2.28 & $\mathrm{Ba}$ & 2.69 ABbc & 2.69 & $\mathrm{ABabc}$ & $2.74 \mathrm{ABb}$ & 2.85 & $\mathrm{ABb}$ & 2.88 & $\mathrm{Ab}$ & $2.97 \mathrm{Ab}$ \\
\hline PI 140471 - 12 & 0.82 & $\mathrm{Ca}$ & 2.07 & $\mathrm{Ba}$ & 2.41 & $\mathrm{ABa}$ & $2.48 \mathrm{ABbc}$ & 2.60 & $\mathrm{Abc}$ & $2.64 \mathrm{Ab}$ & 2.69 & $\mathrm{Ab}$ & 2.72 & $\mathrm{Ab}$ & $2.92 \mathrm{Ab}$ \\
\hline PI 140471 - 13 & 0.68 & $\mathrm{Da}$ & 2.19 & $\mathrm{Ca}$ & 2.49 & $\mathrm{BCa}$ & $2.76 \mathrm{ABCbc}$ & 2.88 & $\mathrm{ABabc}$ & $3.01 \mathrm{ABb}$ & 3.22 & $\mathrm{Ab}$ & 3.25 & $\mathrm{Ab}$ & $3.41 \mathrm{Ab}$ \\
\hline PI 140471 - 15 & 0.51 & $\mathrm{Da}$ & 2.18 & $\mathrm{Ca}$ & 2.53 & $\mathrm{BCa}$ & $2.70 \mathrm{ABCbc}$ & 2.74 & $\mathrm{ABCabc}$ & $2.87 \mathrm{ABb}$ & 3.30 & $\mathrm{Ab}$ & 3.29 & $\mathrm{Ab}$ & $3.28 \mathrm{Ab}$ \\
\hline PI 140471 - 16 & 1.21 & $\mathrm{Ca}$ & 2.32 & $\mathrm{Ba}$ & 2.75 & $\mathrm{ABa}$ & $2.91 \mathrm{ABa}$ & 3.07 & Aab & $3.11 \mathrm{Ab}$ & 3.19 & $\mathrm{Ab}$ & 3.27 & $\mathrm{Ab}$ & $3.27 \mathrm{Ab}$ \\
\hline PI 140471 - 18 & 0.74 & $\mathrm{Ea}$ & 1.83 & $\mathrm{Da}$ & 2.20 & $\mathrm{CDa}$ & $2.49 \mathrm{BCbc}$ & 2.56 & $\mathrm{BCbc}$ & $2.72 \mathrm{BCb}$ & 3.01 & $\mathrm{ABb}$ & 3.47 & $\mathrm{Ab}$ & $3.67 \mathrm{Ab}$ \\
\hline Anôn. 2-01 & 0.31 & $\mathrm{Da}$ & 1.83 & $\mathrm{Ca}$ & 2.28 & $\mathrm{BCa}$ & $2.35 \mathrm{ABCbc}$ & 2.51 & $\mathrm{ABbc}$ & $2.72 \mathrm{Ab}$ & 2.77 & $\mathrm{Ab}$ & 2.77 & $\mathrm{Ab}$ & $3.01 \mathrm{Ab}$ \\
\hline Anôn. ${ }^{\circ}$-02 & 0.93 & $\mathrm{Da}$ & 1.90 & $\mathrm{Ba}$ & 2.56 & $\mathrm{Aa}$ & $2.60 \mathrm{Abc}$ & 2.90 & Aabc & $2.90 \mathrm{Ab}$ & 3.01 & $\mathrm{Ab}$ & 3.06 & $\mathrm{Ab}$ & $3.09 \mathrm{Ab}$ \\
\hline Anôn. 2-04 & 0.75 & $\mathrm{Da}$ & 1.58 & $\mathrm{Ca}$ & 1.95 & $\mathrm{BCa}$ & $2.24 \mathrm{Bbc}$ & 2.24 & $\mathrm{Bbc}$ & $2.43 \mathrm{ABb}$ & 2.43 & $\mathrm{ABb}$ & 2.87 & $\mathrm{Ab}$ & $2.87 \mathrm{Ab}$ \\
\hline Anôn. ${ }^{0} 2-05$ & 1.06 & $\mathrm{Da}$ & 1.81 & $\mathrm{Ca}$ & 2.36 & $\mathrm{BCa}$ & $2.55 \mathrm{ABbc}$ & 2.59 & $\mathrm{ABbc}$ & $2.47 \mathrm{ABb}$ & 2.88 & $\mathrm{ABb}$ & 3.02 & $\mathrm{Ab}$ & $3.08 \mathrm{Ab}$ \\
\hline Anôn. ${ }^{\circ}$-06 & 1.31 & $\mathrm{Da}$ & 1.98 & $\mathrm{Ca}$ & 2.48 & $\mathrm{BCa}$ & $2.73 \mathrm{ABbc}$ & 2.81 & $\mathrm{ABab}$ & $3.14 \mathrm{ABb}$ & 3.22 & $\mathrm{Ab}$ & 3.30 & $\mathrm{Ab}$ & $3.27 \mathrm{Ab}$ \\
\hline Anôn. 2-09 & 0.72 & $\mathrm{Da}$ & 1.89 & $\mathrm{Ca}$ & 2.27 & $\mathrm{BCa}$ & $2.77 \mathrm{ABbc}$ & 2.99 & Aabc & $2.99 \mathrm{Ab}$ & 2.99 & $\mathrm{Ab}$ & 3.24 & $\mathrm{Ab}$ & $3.27 \mathrm{Ab}$ \\
\hline Anôn. ${ }^{\circ}$ - 11 & 1.15 & $\mathrm{Da}$ & 1.95 & $\mathrm{Ca}$ & 2.26 & $\mathrm{BCa}$ & $2.81 \mathrm{ABbc}$ & 2.84 & $\mathrm{ABabc}$ & $3.03 \mathrm{Ab}$ & 3.15 & $\mathrm{Ab}$ & 3.19 & $\mathrm{Ab}$ & $3.26 \mathrm{Ab}$ \\
\hline Anôn. ${ }^{\circ}$ - 12 & 1.11 & & 1.98 & $\mathrm{Da}$ & 2.40 & $\mathrm{CDa}$ & $2.69 \mathrm{BCbc}$ & 2.77 & BCabc & $2.98 \mathrm{ABCb}$ & 2.98 & $\mathrm{ABCb}$ & 3.24 & $\mathrm{ABb}$ & $3.49 \mathrm{Ab}$ \\
\hline Anôn. ${ }^{\circ}$-13 & 1.30 & $\mathrm{Ea}$ & 2.04 & $\mathrm{Ca}$ & 2.28 & $\mathrm{BCa}$ & $2.53 \mathrm{ABCbc}$ & 2.53 & $\mathrm{ABCbc}$ & $2.74 \mathrm{ABb}$ & 2.95 & $\mathrm{ABb}$ & 2.98 & $\mathrm{Ab}$ & $3.10 \mathrm{Ab}$ \\
\hline Anôn. 2-16 & 1.34 & & 2.00 & $\mathrm{Ca}$ & 2.32 & $\mathrm{BCa}$ & $2.67 \mathrm{ABbc}$ & 2.80 & $\mathrm{AB} a b c$ & $2.94 \mathrm{ABb}$ & 2.94 & $\mathrm{Ab}$ & 3.01 & $\mathrm{Ab}$ & $3.04 \mathrm{Ab}$ \\
\hline Anôn. ${ }^{\circ}$-17 & 1.12 & & 1.78 & $\mathrm{Ca}$ & 2.07 & $\mathrm{BCa}$ & $2.63 \mathrm{ABbc}$ & 2.85 & Aabc & $2.88 \mathrm{Ab}$ & 2.97 & $\mathrm{Ab}$ & 3.01 & $\mathrm{Ab}$ & $3.06 \mathrm{Ab}$ \\
\hline Anôn. ${ }^{\circ}$ - 19 & 0.40 & $\mathrm{Ca}$ & 1.85 & $\mathrm{Ba}$ & 2.39 & $\mathrm{ABa}$ & $2.58 \mathrm{Abc}$ & 2.72 & Aabc & $2.86 \mathrm{Ab}$ & 2.94 & $\mathrm{Ab}$ & 2.99 & $\mathrm{Ab}$ & $3.03 \mathrm{Ab}$ \\
\hline Anôn. $2-20$ & 0.66 & $\mathrm{Ea}$ & 1.86 & $\mathrm{Ca}$ & 2.49 & $\mathrm{Ca}$ & $2.69 \mathrm{BCbc}$ & 2.87 & $\mathrm{ABCabc}$ & $2.95 \mathrm{ABCb}$ & 2.99 & $\mathrm{ABCb}$ & 3.23 & $\mathrm{ABb}$ & $3.42 \mathrm{Ab}$ \\
\hline Anôn. ${ }^{\circ}$-21 & 0.68 & $\mathrm{Da}$ & 2.03 & $\mathrm{BCa}$ & 2.24 & $\mathrm{BCa}$ & $2.40 \mathrm{BCbc}$ & 2.57 & $\mathrm{ABCbc}$ & $2.57 \mathrm{ABCb}$ & 2.68 & $\mathrm{ABb}$ & 2.73 & $\mathrm{ABb}$ & $3.16 \mathrm{Ab}$ \\
\hline Anôn. ${ }^{0}$-22 & 1.30 & $\mathrm{Ca}$ & 2.00 & $\mathrm{Ba}$ & 2.12 & $\mathrm{ABa}$ & $2.28 \mathrm{ABbc}$ & 2.43 & $\mathrm{ABbc}$ & $2.56 \mathrm{ABb}$ & 2.60 & $\mathrm{ABb}$ & 2.59 & $\mathrm{Ab}$ & $2.75 \mathrm{Ab}$ \\
\hline
\end{tabular}

${ }^{1}$ The presented results are given original notes.

${ }^{2}$ Means followed by the same capital letter, in the lines, and means followed by the same small letter, in the columns don't differ to each other for the test of Tukey at the level of $5 \%$.

The inheritance of resistance to D. bryoniae in the PI 140471 was reported by Prasad and Norton (1967) as due to a dominant gene, under inoculation in field conditions. Prasad and Norton (1967) used quantitative inheritance criterion to evaluation of the resistance and the grouping of plants resistant or susceptible could be considered an interpretation subject and therefore qualitative inheritance in the PI 140471 was questionable.

This fact was confirmed when the behavior of the
PI 140471 was observed in the experiments of present work. The reaction to the disease using used a quantitative scale of values varied a lot in function of climatic conditions. The inheritance of resistance to $D$. bryoniae in Mi-tang-ting was also studied by Takada (1982) who stated that the generation $F_{1}$, original cross of this variety and susceptible variety were intermediate among the parentals. 
Table 3 - Variance analysis of the resistance of melon progenies inoculated with D. bryoniae in function of evaluation times in plastic tunnel conditions. São Manuel, 1992.

\begin{tabular}{lcc}
\hline \multicolumn{1}{c}{ SV } & DF & MS \\
\hline Blocks & 1 & 0.010 \\
Progenies & 30 & $0.063^{*}$ \\
Error (a) & 30 & 0.025 \\
\hline Parcel & 61 & $7.616^{*}$ \\
Time & 8 & $0.007^{*}$ \\
\hline Time x Progenies & 240 & 0.005 \\
\hline Error (b) & 278 & 9.300 \\
\hline CV (\%) & & \\
\hline
\end{tabular}

* Significant for the test $\mathrm{F}$ at the level of $5 \%$ of probability.

He supposed that two or three resistance genes existed, and the resistance was influenced by screen method of populations and environmental conditions in which it was done.

Considering that the inheritance to $D$. bryoniae in melon could be quantitative, it was tried to test the existent variability in PI 140471 and Anô $n^{\circ} 2$ populations. Through results obtained with evaluations with 45 days, it could be observed that PI 140471 was variable for resistance to disease, while Anô $\mathrm{n}^{\circ} 2$ was uniform. The mean level of resistance of groups of progenies of two populations inspite of difference was very close and they differed a lot with susceptible check, Eldorado 300.

Table 4 - Reaction of melon progenies inoculated with D. bryoniae in function of the evaluation times in plastic tunnel conditions. São Manuel, 1992.

\begin{tabular}{|c|c|c|c|c|c|c|c|c|c|}
\hline \multirow{2}{*}{ Progenies } & \multicolumn{9}{|c|}{ Evaluation Time $(\text { days })^{1,2}$} \\
\hline & 5 & 10 & 15 & 20 & 25 & 30 & 35 & 40 & 45 \\
\hline Eldorado 300 & $0.00 \mathrm{Ea}$ & $0.99 \mathrm{Da}$ & $2.26 \mathrm{Ca}$ & $2.81 \mathrm{BCa}$ & $2.81 \mathrm{BCa}$ & $2.98 \mathrm{ABa}$ & $3.41 \mathrm{ABa}$ & $3.65 \mathrm{ABa}$ & $3.72 \mathrm{~A}$ \\
\hline PI 140471-01 & $0.00 \mathrm{Ca}$ & $1.08 \mathrm{Ba}$ & $2.04 \mathrm{Aa}$ & $2.14 \mathrm{~A} a b$ & $2.19 \mathrm{~A} a b$ & $2.23 \mathrm{~A} \mathrm{ab}$ & $2.53 \mathrm{Ab}$ & $2.48 \mathrm{Ab}$ & $2.48 \mathrm{~A} \mathrm{~b}$ \\
\hline PI 140471-02 & $0.00 \mathrm{Ca}$ & $0.69 \mathrm{Ba}$ & $1.94 \mathrm{Aa}$ & $2.00 \mathrm{~A} \mathrm{~b}$ & $2.09 \mathrm{~A} \mathrm{ab}$ & $2.09 \mathrm{~A} \mathrm{ab}$ & $2.09 \mathrm{~A} \mathrm{~b}$ & $2.09 \mathrm{~A} \mathrm{~b}$ & $2.19 \mathrm{~A} \mathrm{~b}$ \\
\hline PI 140471-03 & $0.00 \mathrm{Ca}$ & $0.39 \mathrm{Ba}$ & $1.94 \mathrm{Aa}$ & $1.93 \mathrm{~A} \mathrm{~b}$ & $2.05 \mathrm{~A} \mathrm{~b}$ & $205 \mathrm{~A} \mathrm{~b}$ & $2.05 \mathrm{~A} \mathrm{~b}$ & $2.09 \mathrm{~A} \mathrm{~b}$ & $2.14 \mathrm{~A} \mathrm{~b}$ \\
\hline PI 140471-04 & $0.00 \mathrm{Da}$ & $0.38 \mathrm{Ca}$ & $1.35 \mathrm{Ba}$ & $2.05 \mathrm{~A} \mathrm{~b}$ & $2.00 \mathrm{~A} \mathrm{~b}$ & $2.09 \mathrm{~A} \mathrm{ab}$ & $2.05 \mathrm{Ab}$ & $2.05 \mathrm{~A} \mathrm{~b}$ & $2.14 \mathrm{~A} \mathrm{~b}$ \\
\hline PI 140471-05 & $0.00 \mathrm{Ca}$ & $0.51 \mathrm{Ba}$ & $1.50 \mathrm{Aa}$ & $1.96 \mathrm{~A} \mathrm{~b}$ & $2.04 \mathrm{~A} \mathrm{~b}$ & $2.09 \mathrm{~A} \mathrm{ab}$ & $2.09 \mathrm{~A} \mathrm{~b}$ & $2.15 \mathrm{Ab}$ & $2.15 \mathrm{~A} \mathrm{~b}$ \\
\hline PI 140471-07 & $0.00 \mathrm{Ca}$ & $0.31 \mathrm{Ba}$ & $1.77 \mathrm{Aa}$ & $1.93 \mathrm{~A} \mathrm{~b}$ & $2.19 \mathrm{~A} a b$ & $2.14 \mathrm{~A} \mathrm{ab}$ & $2.19 \mathrm{~A} \mathrm{~b}$ & $2.19 \mathrm{~A} \mathrm{~b}$ & $2.14 \mathrm{~A} \mathrm{~b}$ \\
\hline PI 140471 - 10 & $0.00 \mathrm{Ca}$ & $0.41 \mathrm{Ba}$ & $2.08 \mathrm{Aa}$ & $2.13 \mathrm{~A} \mathrm{ab}$ & $2.23 \mathrm{~A} \mathrm{ab}$ & $2.23 \mathrm{~A} \mathrm{ab}$ & $2.48 \mathrm{~A} \mathrm{~b}$ & $2.48 \mathrm{~A} \mathrm{~b}$ & $2.53 \mathrm{~A} \mathrm{~b}$ \\
\hline PI 140471-11 & $0.00 \mathrm{Ca}$ & $0.41 \mathrm{Ba}$ & $1.69 \mathrm{Aa}$ & $2.16 \mathrm{~A} a b$ & $2.13 \mathrm{~A} a b$ & $2.30 \mathrm{~A} \mathrm{ab}$ & $2.30 \mathrm{~A} \mathrm{~b}$ & $2.35 \mathrm{~A} \mathrm{~b}$ & $2.35 \mathrm{~A} \mathrm{~b}$ \\
\hline PI 140471- 12 & $0.00 \mathrm{Da}$ & $0.48 \mathrm{Ca}$ & $1.31 \mathrm{Ba}$ & $2.04 \mathrm{~A} \mathrm{~b}$ & $2.14 \mathrm{~A} a b$ & $2.05 \mathrm{~A} \mathrm{~b}$ & $2.09 \mathrm{~A} \mathrm{~b}$ & $2.14 \mathrm{Ab}$ & $2.14 \mathrm{~A} \mathrm{~b}$ \\
\hline PI 140471-13 & $0.00 \mathrm{Da}$ & $0.88 \mathrm{Ca}$ & $1.84 \mathrm{Ba}$ & $2.28 \mathrm{~A} a b$ & $2.43 \mathrm{~A} a b$ & $2.48 \mathrm{~A} \mathrm{ab}$ & $2.48 \mathrm{~A} \mathrm{~b}$ & $2.72 \mathrm{~A} \mathrm{~b}$ & $2.77 \mathrm{~A} \mathrm{a}$ \\
\hline PI 140471-15 & $0.00 \mathrm{Ca}$ & $0.19 \mathrm{Ba}$ & $1.71 \mathrm{Aa}$ & $2.19 \mathrm{~A} \mathrm{ab}$ & $2.23 \mathrm{~A} \mathrm{ab}$ & $2.33 \mathrm{~A} \mathrm{ab}$ & $2.38 \mathrm{Ab}$ & $2.38 \mathrm{~A} \mathrm{~b}$ & $2.38 \mathrm{~A} \mathrm{~b}$ \\
\hline PI 140471- 16 & $0.00 \mathrm{Ca}$ & $0.08 \mathrm{Ca}$ & $1.51 \mathrm{Ba}$ & $2.23 \mathrm{~A} \mathrm{ab}$ & $2.23 \mathrm{~A} \mathrm{ab}$ & $2.23 \mathrm{~A} \mathrm{ab}$ & $2.28 \mathrm{~A} \mathrm{~b}$ & $2.28 \mathrm{~A} \mathrm{~b}$ & $2.28 \mathrm{~A} \mathrm{~b}$ \\
\hline PI 140471 - 18 & $0.00 \mathrm{Da}$ & $0.66 \mathrm{Ca}$ & $1.74 \mathrm{Ba}$ & $2.23 \mathrm{AB} a b$ & $2.23 \mathrm{ABab}$ & $2.28 \mathrm{ABab}$ & $2.33 \mathrm{ABb}$ & $2.33 \mathrm{ABb}$ & $251 \mathrm{~A} \mathrm{~b}$ \\
\hline Anôn.2-01 & $0.00 \mathrm{Da}$ & $0.72 \mathrm{Ca}$ & $1.47 \mathrm{Ba}$ & $2.00 \mathrm{ABb}$ & $2.00 \mathrm{ABb}$ & $2.05 \mathrm{AB} b$ & $2.05 \mathrm{ABb}$ & $2.17 \mathrm{Ab}$ & $2.17 \mathrm{~A} \mathrm{~b}$ \\
\hline Anôn. ${ }^{\circ} 2-02$ & $0.00 \mathrm{Ca}$ & $0.39 \mathrm{Ba}$ & $2.07 \mathrm{Aa}$ & $2.19 \mathrm{~A} a b$ & $2.23 \mathrm{~A} \mathrm{ab}$ & $2.23 \mathrm{~A} \mathrm{ab}$ & $2.23 \mathrm{~A} \mathrm{~b}$ & $2.28 \mathrm{~A} \mathrm{~b}$ & $2.28 \mathrm{~A} \mathrm{~b}$ \\
\hline Anôn.'2-03 & $0.00 \mathrm{Ca}$ & $0.43 \mathrm{Ba}$ & $1.46 \mathrm{Aa}$ & $1.92 \mathrm{~A} \mathrm{~b}$ & $200 \mathrm{~A} \mathrm{~b}$ & $2.00 \mathrm{~A} \mathrm{~b}$ & $2.06 \mathrm{~A} \mathrm{~b}$ & $2.06 \mathrm{~A} \mathrm{~b}$ & $2.06 \mathrm{~A} \mathrm{~b}$ \\
\hline Anôn. ${ }^{\circ}$ 2-04 & $0.00 \mathrm{Ca}$ & $0.16 \mathrm{Ba}$ & $1.92 \mathrm{Aa}$ & $2.00 \mathrm{~A} \mathrm{~b}$ & $2.00 \mathrm{~A} \mathrm{~b}$ & $2.00 \mathrm{~A} \mathrm{~b}$ & $2.00 \mathrm{~A} \mathrm{~b}$ & $2.00 \mathrm{~A} \mathrm{~b}$ & $2.00 \mathrm{~A} \mathrm{~b}$ \\
\hline Anôn. 2-05 & $0.00 \mathrm{Ca}$ & $0.51 \mathrm{Ba}$ & $2.00 \mathrm{Aa}$ & $2.19 \mathrm{~A} a b$ & $2.19 \mathrm{~A}$ ab & $2.19 \mathrm{~A} \mathrm{ab}$ & $2.19 \mathrm{~A} \mathrm{~b}$ & $2.33 \mathrm{~A} \mathrm{~b}$ & $2.48 \mathrm{~A} \mathrm{~b}$ \\
\hline
\end{tabular}




\begin{tabular}{|c|c|c|c|c|c|c|c|c|c|}
\hline Anôn. ${ }^{\circ}$ 2-06 & $0.00 \mathrm{Da}$ & $0.86 \mathrm{Ca}$ & $1.52 \mathrm{Ba}$ & $2.19 \mathrm{~A} a b$ & $2.19 \mathrm{~A} \mathrm{ab}$ & $2.43 \mathrm{~A} \mathrm{~b}$ & $2.53 \mathrm{~A} \mathrm{ab}$ & $2.53 \mathrm{~A} \mathrm{~b}$ & $2.53 \mathrm{~A} \mathrm{~b}$ \\
\hline Anôn.'²-09 & $0.00 \mathrm{Ca}$ & $0.51 \mathrm{Ba}$ & $2.05 \mathrm{Aa}$ & $2.05 \mathrm{~A} \mathrm{~b}$ & $2.05 \mathrm{~A} \mathrm{~b}$ & $2.05 \mathrm{~A} \mathrm{~b}$ & $2.05 \mathrm{~A} \mathrm{~b}$ & $2.05 \mathrm{~A} \mathrm{~b}$ & $2.05 \mathrm{~A} \mathrm{~b}$ \\
\hline Anôn.' 2-11 & $0.00 \mathrm{Ca}$ & $0.88 \mathrm{Ba}$ & $1.77 \mathrm{Aa}$ & $2.05 \mathrm{~A} \mathrm{~b}$ & $2.05 \mathrm{~A} \mathrm{~b}$ & $2.05 \mathrm{~A} \mathrm{~b}$ & $2.05 \mathrm{~A} \mathrm{~b}$ & $2.05 \mathrm{~A} \mathrm{~b}$ & $2.14 \mathrm{~A} \mathrm{~b}$ \\
\hline Anôn. ${ }^{\circ}$ - 12 & $0.00 \mathrm{Da}$ & $0.47 \mathrm{Ca}$ & $1.44 \mathrm{Ba}$ & $2.02 \mathrm{~A} \mathrm{~b}$ & $2.13 \mathrm{~A} \mathrm{ab}$ & $2.13 \mathrm{~A} a b$ & $2.13 \mathrm{Ab}$ & $2.13 \mathrm{~A} \mathrm{~b}$ & $2.13 \mathrm{~A} \mathrm{~b}$ \\
\hline Anôn. ${ }^{\circ}$-13 & $0.00 \mathrm{Ca}$ & $0.34 \mathrm{Ba}$ & $2.00 \mathrm{Aa}$ & $2.09 \mathrm{~A} \mathrm{ab}$ & $2.09 \mathrm{~A} \mathrm{ab}$ & $2.09 \mathrm{~A} a b$ & $2.09 \mathrm{~A} \mathrm{~b}$ & $2.09 \mathrm{~A} \mathrm{~b}$ & $2.14 \mathrm{~A} \mathrm{~b}$ \\
\hline Anôn. ${ }^{0} 2-16$ & $0.00 \mathrm{Ca}$ & $0.63 \mathrm{Ba}$ & $1.60 \mathrm{Aa}$ & $2.05 \mathrm{~A} \mathrm{~b}$ & $2.05 \mathrm{~A} \mathrm{~b}$ & $2.05 \mathrm{~A} \mathrm{~b}$ & $2.05 \mathrm{Ab}$ & $2.05 \mathrm{~A} \mathrm{~b}$ & $2.05 \mathrm{~A} \mathrm{~b}$ \\
\hline Anôn. ${ }^{\circ}$-17 & $0.00 \mathrm{Ca}$ & $0.42 \mathrm{Ba}$ & $1.79 \mathrm{Aa}$ & $2.00 \mathrm{~A} \mathrm{~b}$ & $2.00 \mathrm{~A} \mathrm{~b}$ & $2.00 \mathrm{~A} \mathrm{~b}$ & $2.00 \mathrm{~A} \mathrm{~b}$ & $2.00 \mathrm{~A} \mathrm{~b}$ & $2.00 \mathrm{~A} \mathrm{~b}$ \\
\hline Anôn.'2-18 & $0.00 \mathrm{Ca}$ & $0.23 \mathrm{Ca}$ & $1.16 \mathrm{Ba}$ & $1.86 \mathrm{~A} \mathrm{~b}$ & $2.00 \mathrm{~A} \mathrm{~b}$ & $1.86 \mathrm{~A} \mathrm{~b}$ & $2.00 \mathrm{~A} \mathrm{~b}$ & $2.05 \mathrm{~A} \mathrm{~b}$ & $2.05 \mathrm{~A} \mathrm{~b}$ \\
\hline Anôn. '2-19 & $0.00 \mathrm{Ca}$ & $0.10 \mathrm{Ba}$ & $1.88 \mathrm{Aa}$ & $2.05 \mathrm{~A} \mathrm{~b}$ & $2.09 \mathrm{~A} \mathrm{ab}$ & $2.09 \mathrm{~A} \mathrm{ab}$ & $2.09 \mathrm{~A} \mathrm{~b}$ & $2.09 \mathrm{~A} \mathrm{~b}$ & $2.19 \mathrm{~A} \mathrm{~b}$ \\
\hline Anôn. ${ }^{\circ} 2-20$ & $0.00 \mathrm{Ba}$ & $0.34 \mathrm{Ba}$ & $2.05 \mathrm{Aa}$ & $2.00 \mathrm{~A} \mathrm{~b}$ & $2.05 \mathrm{~A} \mathrm{~b}$ & $2.05 \mathrm{~A} \mathrm{~b}$ & $2.05 \mathrm{Ab}$ & $2.05 \mathrm{~A} \mathrm{~b}$ & $2.14 \mathrm{~A} \mathrm{~b}$ \\
\hline Anôn. ${ }^{0} 2-21$ & $0.00 \mathrm{Da}$ & $0.45 \mathrm{Ca}$ & $1.06 \mathrm{Ba}$ & $2.09 \mathrm{~A} \mathrm{ab}$ & $2.09 \mathrm{~A} \mathrm{ab}$ & $2.09 \mathrm{~A} \mathrm{ab}$ & $2.09 \mathrm{~A} \mathrm{~b}$ & $2.09 \mathrm{~A} \mathrm{~b}$ & $2.09 \mathrm{~A} \mathrm{~b}$ \\
\hline Anôn. 2-22 & $0.00 \mathrm{Ca}$ & $0.17 \mathrm{Ba}$ & $1.62 \mathrm{Aa}$ & $2.00 \mathrm{~A} \mathrm{~b}$ & $2.00 \mathrm{~A} \mathrm{~b}$ & $2.00 \mathrm{~A} \mathrm{~b}$ & $2.00 \mathrm{~A} \mathrm{~b}$ & $2.29 \mathrm{~A} \mathrm{~b}$ & $2.29 \mathrm{~A} \mathrm{~b}$ \\
\hline
\end{tabular}

\footnotetext{
${ }^{1}$ The presented results are given original notes.

${ }^{2}$ Means followed by the same capital letter, in the lines, and means followed by the same small letter, in the columns, don't differ to each other for the test of Tukey at the level of 5\%.
}

Table 5 - Variance analysis of the resistance of melon progenies appraised 45 days after the inoculation with $D$. bryoniae in conditions of plastic tunnel, São Manuel, 1992.

\begin{tabular}{lrc}
\hline \multicolumn{1}{c|}{ Sources of Variation } & DF & MS \\
\hline Blocks & 1 & 0.072 \\
\hline Between progenies & 29 & $0.062^{*}$ \\
\hline Between progenies PI 140471 $^{\circ}$ & 12 & $0.069^{*}$ \\
Between progenies Anô n. $^{2}$ & 16 & 0.040 \\
PI 140471 vs. Anô n. ${ }^{\circ}$ & 1 & $0.338^{*}$ \\
\hline Error & 29 & 0.033 \\
Within progenies & 516 & 0.015 \\
\hline
\end{tabular}

* Significant for the test $\mathrm{F}$ at the level of $5 \%$ of probability.

From point of view of improvement Anô $\mathrm{n}^{\circ} 2$ could be most appropriate population for use. This variety has resistance to gummy stem blight, CMV (Cucumber Mosaic Virus), downy mildew and powdery mildew. Moreover it has having desirable commercial characteristics such as appropriate size of fruit, green pulp thickens and high tenor of soluble solids.

\section{ACKNOWLEDGEMENTS}

The authors wish to thank Dr. Chukichi Kurozawa for the Departamento de Produção Vegetal - Setor de Defesa Fitossanitária, FCA/UNESP, Campus de Botucatu, for the valuable contributions during the development of this work and the CAPES for the master scholarship to the CYT.

\section{RESUMO}

O presente trabalho objetiva avaliar populações de melão para resistência ao Crestamento Gomoso do Caule, causado por Didymella bryoniae, para uso em programas de melhoramento em condições de casa de vegetação e túnel plástico. Foram avaliadas populações de meio irmãos das introduções PI 140471 e Anô n. ${ }^{\circ} 2$ e a testemunha suscetível Eldorado 300. As plantas foram inoculadas aos 45 dias por picada de agulha na axila da terceira e da quarta folhas verdadeiras seguida da aplicação de $0,05 \mathrm{ml}$ de suspensão de esporos a uma concentração de $5,0 \times 10^{5}$ esporos $/ \mathrm{ml}$, e sob condições de túnel plástico. A avaliação da resistência foi efetuada através de uma escala de notas variando de zero a cinco até as plantas atingirem 90 dias de idade. As progênies de meio irmãos de PI 140471 e de Anô n. ${ }^{\circ} 2$ mostraram-se superiores e diferiram 
significativamente da testemunha Eldorado 300 pelo Teste de Tukey a nível de 5\%. A cultivar Anô n. ${ }^{\circ} 2$, por possuir resistência múltipla a doenças e características comerciais desejáveis, constitui-se na melhor fonte de resistência a ser utilizada em programas de melhoramento de melão.

\section{REFERENCES}

Chiu, W. F. and Walker, J. C. (1949), Physiology and pathogenicity of cucurbit black-rot fungus. Journal of Agricultural Research, 78, 589-615.

Malathrakis, N. E. and Vakalounakis, D. J. (1983), Resistance to benzinidazole fungicides in the Gummy stem blight pathogen Didymella bryoniae on cucurbits. Plant Pathology, 32, 395-399.

Norton, J. D. and Prasad, K. (1965), Incorporation of recently discovered resistance to gummy stem blight, Mycosphaerella melonis (Pass.) Chiu and Walker, in to eduble types of muskmelon. Proceedings of Southern Agricultural Workers, 62, 154.

Pimentel Gomes, F. (1987), Curso de Estatística Experimental. Piracicaba : Nobel. 467 pp.

Prasad, K. and Norton, J. D. (1967), Inheritance of resistance to Mycosphaerellha citrullina in muskmelon. Proceedings of American Society for Horticultural Science, 91, 396-400.

Sowell, G.; Prasad, K. and Norton, J. D. (1966), Resistance of Cucumis melo introductions to Mycosphaerella citrulina. Plant Disease Reporter, 50, 661-663.

Sowell, G. (1981), Additional sources of resistance to gummy stem blight of muskmelon. Plant Disease, 65, 253-254.

Takada, K.; Kanazawa, K. and Takatuka, K. (1980), Studies on the breeding of melon resistance to gummy stem blight. I. Differences and regional distribution of varietal resistance. Bulletin of Vegetable and Ornamental Research Station. Serie A, 7, 1-11.

Takada, K. (1982), Breeding methods of diseas e-resistance of melon, and development of new lines with combined resistance. JARQ, 16, 94-104.
Takada, K. (1983), Breeding and characteristics of disease resistant melon varieties (lines "Anô $\mathrm{n}^{\circ} 1, \mathrm{n}^{\mathbf{0}} 2$ e $\mathrm{n}^{\mathrm{o}} 3$ "). Bulletin of Vegetable and Ornamental Research Station. Serie A, 11, 1-11.

Zhang, Y. P.; Kyle, M.; Anagnostou, K. and Zitter, T. A. (1997), Screening melon (Cucumis melo) for resistance to gummy stem blight in the greenhouse and field. HortScience, 32, 117-121.

Zuniga, T. L.; Jantz, J. P.; Zitter, T. A. and Jahn, M. K. (1999), Monogenic dominant resistance to gummy stem blight in two melon (Cucumis melo) accessions. Plant Disease, 83, 1105-1107.

Received: April 16, 2002; Revised: July 25, 2002; Accepted: March 13, 2003. 